\title{
UM ESTUDO SOBRE A APLICAÇÃO DA GESTÃO DO CONHECIMENTO NA ADMINISTRAÇÃO PÚBLICA
}

\author{
Pablo Machado Amorim (UFF) pabloamorim.cefet@gmail.com \\ Stella Regina Reis da Costa (UFF) stellare@ig.com.br
}

\section{Resumo}

Ante o cenário atual, onde o nível de exigência do cidadão para a prestação dos serviços públicos é gradativamente elevado, além da crise financeira que vem se alastrando, gerando restrições orçamentárias às organizações, é incabível manterem-se as velhas práticas ineficientes na Administração Pública. Neste sentido, a gestão do conhecimento se torna um fator de suma importância para as organizações, uma vez que a geração e a disseminação do conhecimento é peça fundamental no sucesso de qualquer organização que se espelhe na eficiência como norteador de seus trabalhos. O objetivo desse trabalho é apresentar um levantamento conceitual e teórico, realizando uma revisão da literatura pertinente que fundamentará a presente pesquisa. A pesquisa foi elaborada por meio de um levantamento bibliográfico, onde se abrangeu artigos nacionais e internacionais. $\mathrm{O}$ artigo pode ajudar no entendimento dos aspectos teóricos sobre administração pública, eficiência, eficácia e de como a gestão do conhecimento pode contribuir para a melhoria dos serviços públicos colocados à disposição do cidadão. Foi possível concluir que com a gestão do conhecimento é possível atingir a disseminação do conhecimento institucional, gerando maior eficiência, redução de ruídos na comunicação e trabalho uniforme entre todos os funcionários, consequentemente aumentando a satisfação daqueles que utilizam o serviço.

Palavras chave: Gestão do conhecimento; Administração Pública; Eficiência. 


\section{Introdução}

Partindo da premissa que a administração pública tem por objetivo acompanhar as transformações da sociedade e das suas demandas, visando aumentar a satisfação dos interessados pelos serviços. Nesse sentido a gestão do conhecimento, segundo Pardo et al. (2013), constitui-se como um conjunto de atividades que são realizadas com finalidade de utilizar, compartilhar e desenvolver o conhecimento de uma organização.

De acordo com Juárez e Cervantes (2012) a sociedade em geral clama por mais informações por parte da administração pública desejando que novas formas de comunicação e interação sejam desenvolvidas para que se possa garantir o acesso à informação. Essa aspiração da sociedade está diretamente ligada ao desenvolvimento da sociedade do conhecimento, que tem como compromisso os seguintes aspectos:

- Transparência e eficácia na gestão pública;

- Produtividade nas organizações públicas;

- Capacitação na formação dos gestores e funcionários públicos;

- Melhora e variedade na oferta de serviços públicos de qualidade.

Os autores ainda ensinam que "a gestão do conhecimento resulta numa ferramenta de grande transcendência para as organizações públicas, cujo maior capital reside nas pessoas que a integram e nos conhecimentos, tanto fáticos como procedimentais".

Segundo Pardo et al. (2013) as organizações públicas são "grandes produtores e consumidores de conhecimento", devendo, dessa forma, terem especial atenção a dois importantes aspectos:

- Serem eficientes na arrecadação e aplicação dos recursos;

- Melhorarem a capacidade de vida de seus cidadãos mediante a prestação dos serviços.

Dessa forma, a gestão do conhecimento na Administração Pública faz referência ao conhecimento público que a compõe e se bem articulada na gestão do conhecimento poderão fazer melhor uso dos recursos, servindo de alavanca para o sucesso administrativo (BEM; PRADO; DELFINO, 2013).

Não é raro encontrar servidores públicos com relatos quanto à forma como tiveram acesso ao conhecimento das funções que iriam executar no seu dia-a-dia; "foi me passado assim"; "desde que eu entrei que é feito assim"; "o chefe que disse que era desse jeito". De acordo com esses relatos e pela falta de manuais de procedimentos e de instruções de trabalho, 
percebe-se que a administração pública sofre muito com ruído na comunicação e em falta de padronização nos seus processos.

O objetivo desse trabalho é apresentar um levantamento conceitual e teórico, realizando uma revisão da literatura pertinente à gestão do conhecimento com foco na administração pública com a intenção de demonstrar que se é possível a melhoria dos serviços prestados, tornando o serviço público mais eficiente e eficaz.

\section{Metodologia}

A revisão da literatura se baseia em pesquisa qualitativa e explicativa através de técnica indireta. Para o embasamento teórico dessa pesquisa e visando levantar a literatura mais relevante e atualizada com relação direta ao problema de pesquisa, foram realizadas investigações em bases de dados conceituadas no meio acadêmico versando sobre a temática central deste trabalho.

As bases de dados utilizadas nesta investigação foram Web of Science e SCOPUS, ambas acessadas através no Portal de Periódicos da Capes, além de livros, artigos científicos e periódicos nacionais e estrangeiros sendo utilizada a rede mundial de computadores, em sítios de universidades e centros de pesquisas renomados.

Para a realização adequada da pesquisa, foi empregada a técnica de busca por meio de palavras-chave utilizando o operador lógico booleano "AND" para associação de palavras e/ou expressões, optando-se pela utilização dos seguintes termos combinados:

- "Gestão do conhecimento" and "administração pública";

- "knowledge management" and "public administration".

Após análise e leitura dos artigos selecionados chegou-se a um total de trinta e cinco documentos, entre artigos nacionais e internacionais, livros, dissertações, leis entre outros conforme tabela 1 a seguir. 
Tabela 1: Documentos pesquisados

\begin{tabular}{|l|c|c|}
\hline \multicolumn{1}{|c|}{ TIPO } & QUANTIDADE & PERCENTUAL \\
\hline ARTIGOS INTERNACIONAIS & 13 & $37,1 \%$ \\
\hline ARTIGOS NACIONAIS & 12 & $34,3 \%$ \\
\hline LIVROS & 5 & $14,3 \%$ \\
\hline DISSERTAÇÃO & 1 & $2,9 \%$ \\
\hline OUTROS & 4 & $11,4 \%$ \\
\hline TOTAL & 35 & $100 \%$ \\
\hline
\end{tabular}

Fonte: Elaboração própria

Dessa forma pode-se constatar que foram realizadas pesquisas em artigos internacionais e nacionais em bases de dados de grande relevância, o que contribui para a credibilidade e relevância do estudo bibliográfico.

\section{A aplicação da gestão do conhecimento na Administração Pública}

\subsection{Gestão do conhecimento}

No processo de gestão do conhecimento, torna-se fundamental a utilização de três pilares básicos: recursos humanos, processos organizacionais e tecnologias. Os autores ainda destacam que para correta implementação da Gestão do conhecimento é fundamental a utilização dos processos que realmente estejam em execução dentro da organização (CEPELOVA e KORENOVA 2017).

Os servidores públicos têm que aprender a executar os processos dos quais são responsáveis. Esse aprendizado pode ser realizado através de treinamentos, orientação oral, ou por meio de estudo de toda a documentação (CICCIO; MARRELLA; RUSSO, 2014). Para Novoa (2018), uma vez que muitas das operações realizadas em um processo são feitas sem intervenção humana, é de suma importância o envolvimento, o treinamento e o estímulo para melhoria dos profissionais envolvidos com a gestão dos processos.

Reforçando tal entendimento, Aranha (2015) relata a importância que tem a gestão por competências para que as instituições possam conhecer seus pontos fortes e os pontos fracos, passando pelo conhecimento das competências institucionais até as individuais dos servidores. Nesse momento, destaca-se a importância da educação corporativa, que se enquadra como uma das possibilidades para melhoria dos pontos fracos. 
De acordo com Cepelova e Korenova (2017), a teoria tratando de questões de gestão do conhecimento afirma que uma das condições necessárias para a aplicação do modelo de gestão do conhecimento nas organizações é o gerenciamento de processos organizacionais.

Segundo Batista (2012), na gestão do conhecimento voltada para o setor público é necessário incluir os processos de identificação, criação, armazenamento e aplicação do conhecimento, com aprendizagem e inovação. $\mathrm{O}$ autor ainda relata que a gestão do conhecimento gera qualidade, eficiência, efetividade e desenvolvimento econômico e social, contribuindo para a legalidade, impessoalidade, moralidade e publicidade na administração pública.

\subsection{Administração pública}

Tendo como base que o propósito da administração pública é a maximização do bem-estar da sociedade e a otimização dos recursos públicos, é de fundamental importância a definição de estratégias bem delineadas pelos gestores públicos para que se possam atender às demandas da população, usando de forma eficiente os recursos disponíveis e que se tenha o maior número de pessoas atendidas e sucesso no desenvolvimento socioeconômico (ALONSO; CLIFTON; DÍAZ-FUENTES, 2015).

A administração pública tem por obrigação a prestação de serviços aos cidadãos de acordo com as legislações vigentes, para isso, faz-se necessária a definição de procedimentos específicos de forma que os servidores possuam base para orientação e passem a prestar os serviços da melhor forma possível (FERRARI et al., 2018).

Importante frisar que a administração pública é a responsável por implementar as políticas públicas e de atender às demandas dos cidadãos (NOVOA, 2018). As reformas pelas quais o país passou nos últimos anos tendem para uma administração mais participativa, eficiente e integrada em rede, de acordo com Paes de Paula (2005).

O modelo de administração pública gerencial foi proposto para transferir ao setor público conhecimentos e práticas do setor privado (PAES DE PAULA, 2005). O principal objetivo da administração pública gerencial é o aumento da qualidade e a redução de custos. Bresser (2008) ainda complementa que esse tipo de modelo é orientado a resultados e para que se aumente o nível de governança no Estado, gerando eficiência e efetividade aos órgãos públicos. 
Nesse sentido e para que isso ocorra, há a necessidade da implantação de programas voltados à eficiência no serviço público, além da possibilidade de responsabilização de seus agentes. Miranda e Kempfer (2018) colocam a administração pública gerencial como sendo uma forma de reestabelecer a relação da sociedade com o Estado, levando à administração pública novas formas de administrar, tal como na iniciativa privada, gerando produtividade e especialização do serviço.

De acordo com Fadul e Silva (2008) a administração pública gerencial é orientada pela meritocracia e pelo profissionalismo. Os autores ainda complementam que nesse modelo há séria contribuição para o aumento da igualdade quanto ao acesso aos serviços públicos colocados à disposição, mas para que isso se torne possível é necessário planejamento estratégico e um maior grau de autonomia gerencial, elaboração de indicadores de desempenho e avaliação dos resultados.

\subsection{Gestão do Conhecimento na Administração Pública}

A gestão do conhecimento com aplicação prática na Administração Pública tem com intenção contribuir para a melhoria contínua da qualidade no serviço público prestado à sociedade. Fresneda e Gonçalves (2007) relatam que a utilização da gestão do conhecimento pode ser entendida como uma estratégia para a melhoria do desempenho e do relacionamento interno e externo das organizações do setor público, uma vez que se busca a efetividade dos serviços e a otimização dos processos, eliminando redundância, mapeando e compartilhando o conhecimento organizacional (BOTELHO, 2009).

A razão pela qual a administração pública e a gestão do conhecimento se relacionam é muito bem tratada por Angelis (2011), onde o autor relata turbulência com necessidade de adaptação e resolução de problemas, além de mudanças significativas em tecnologia da informação e comunicação.

Medir o nível que a gestão do conhecimento se encontra dentro das organizações se dá pelo ato do aprimoramento constante nos processos de criação, identificação, armazenamento, compartilhamento e aplicação dos conhecimentos organizacionais, contribuindo para que a memória organizacional não se perca com o passar dos anos (SANTOS; BASTOS, 2019). Os autores ainda relatam que o nível de maturidade de uma organização é um dos passos para o aprimoramento das técnicas utilizadas e também para o avanço necessário ás instituições, com vistas à melhoria contínua dos processos administrativos, como demonstrado na figura 1. 
Figura 1: Relação: Administração Pública / Gestão do Conhecimento / Maturidade GC

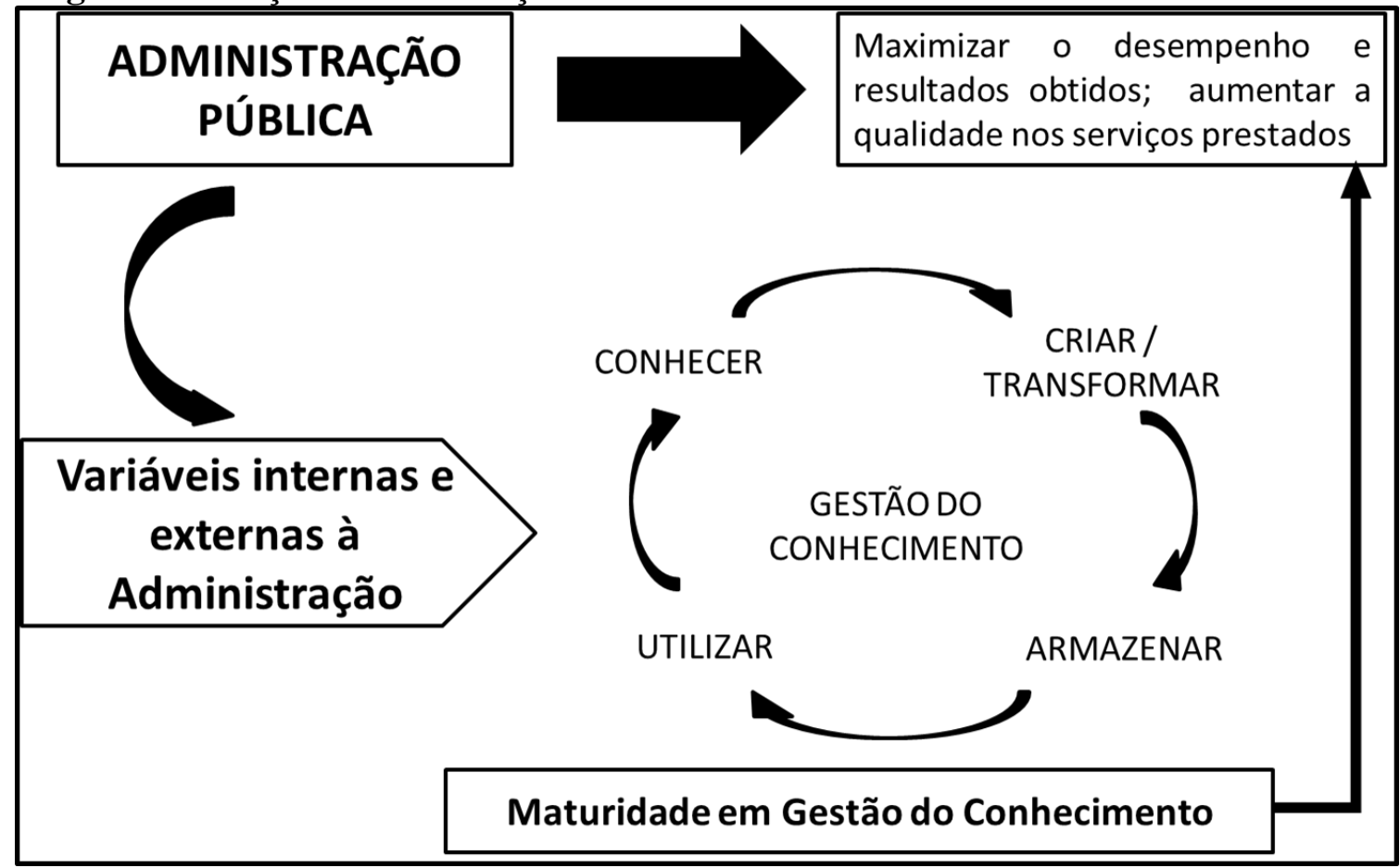

Fonte: Santos e Bastos (2019)

\subsection{Eficiência no setor público}

Chiechelski (2005) afirma que a eficiência no setor público relaciona-se à capacidade do Estado de prover bens e serviços aplicados à sociedade. Com o objetivo de maximização dos resultados e otimização dos recursos, a eficiência na administração pública vem ganhando cada vez mais destaque, tendo sido a mesma consolidada pela Emenda Constitucional nº19/1998 como sendo um dos princípios da administração pública no Brasil, com a finalidade de aumento da qualidade dos serviços prestados (STASTNA; GREGOR, 2011; ADAM; DELIS; KAMMAS, 2011).

A administração pública tem o dever de tomar suas decisões para que se possam atingir os objetivos pretendidos de forma a reduzir as falhas, tempo e recurso, atribuindo aos agentes públicos o dever de realizar suas atribuições com presteza, perfeição e rendimento funcional (MEIRELLES, 1998; DI PIETRO 2012).

Ferrari et al. (2018) ainda inserem a comunicação como sendo um dos caminhos para a eficiência. $\mathrm{O}$ autor relata que a comunicação efetiva do conhecimento dos processos de uma organização é uma necessidade para que qualquer organização estruturada chegue à eficiência. 
Para Lima (2014), apesar de haver semelhanças morfológicas entre os termos eficiência e eficácia seus significados são distintos. A eficiência está diretamente ligada ao tempo de execução da tarefa, ao esforço aplicado para sua solução e o custo dispendido para alcançar o objetivo, enquanto que a eficácia relaciona-se com o atingimento do resultado esperado. Por Jacobsen (2009), gerir com eficácia seria atingir os objetivos planejados, mas gerir com eficiência já implica na utilização correta dos recursos disponíveis.

Outro conceito incorporado pela literatura, segundo Castro (2006) é o de efetividade, sendo o mesmo ainda mais complexo que os conceitos de eficiência e eficácia. Para o autor, o conceito enquanto utilizado pela administração pública tem o objetivo de medir quanto os resultados de determinada ação geram benefícios reais à sociedade. Tal termo é muito mais abrangente e complexo que a eficácia, visto que esta indica apenas se o objetivo foi atingido ou não, a efetividade demonstra quais foram as melhorias geradas à população atendida.

Apesar do desafio de se melhorar os processos e reduzir a burocracia, ainda se percebe a falta de meritocracia nas instituições públicas brasileiras, além da resistência à mudança, o que muitas vezes impede o funcionamento adequado da máquina pública (ANDERSON et al., 2018).

\subsection{Inovação na administração pública}

Em razão da escassez dos recursos públicos para atendimento às demandas sociais nos últimos anos, tem havido uma necessidade por incentivos às ações inovadoras no serviço público diante do aparato governamental. Segundo Mulgan e Albury (2003), a inovação torna-se uma importante ferramenta para o serviço público uma vez que cria e implementa novos processos, produtos, serviços e métodos de entrega, resultando melhorias significativas em busca da eficiência, da eficácia e da qualidade quanto aos resultados.

A inserção de práticas inovadoras na administração pública tem a intenção de aumentar a eficiência e a qualidade dos bens e serviços públicos. O interesse pela inovação no setor público vem aumentando após o início das ações que premiam instituições governamentais, como por exemplo, o Concurso Inovação da Gestão Pública Federal, promovido pela Escola Nacional de Administração Pública (ENAP).

Em se tratando do ponto de vista da inovação, a utilização das TICs beneficia a todos os interessados, uma vez que os cidadãos lucram com o aumento da eficiência e da eficácia no uso dos recursos públicos disponíveis; os servidores públicos beneficiam-se com o aumento 
da produtividade da unidade organizacional onde trabalham e pela confiança no desempenho de suas funções; os gestores públicos ganham com a modernização administrativa dos órgãos e a administração pública ganha em efetividade na aplicação de suas tarefas (COUTO et al., 2018).

\subsection{Gestão do Conhecimento e a Integração com a Inovação}

Fazer uma gestão eficiente das competências organizacionais pode ser considerado um fator chave para o sucesso, porém ainda constitui-se uma lacuna, como o conhecimento pode ser combinado e convertido em ambientes inovadores. Nonaka e Takeuchi (1997) ensinam que a produção do conhecimento é constituída por quatro fases: Socialização, Externalização, Combinação e Internalização, como bem demonstrado na figura 2 e explicado em seguida.

Figura 2: Fases da produção do conhecimento

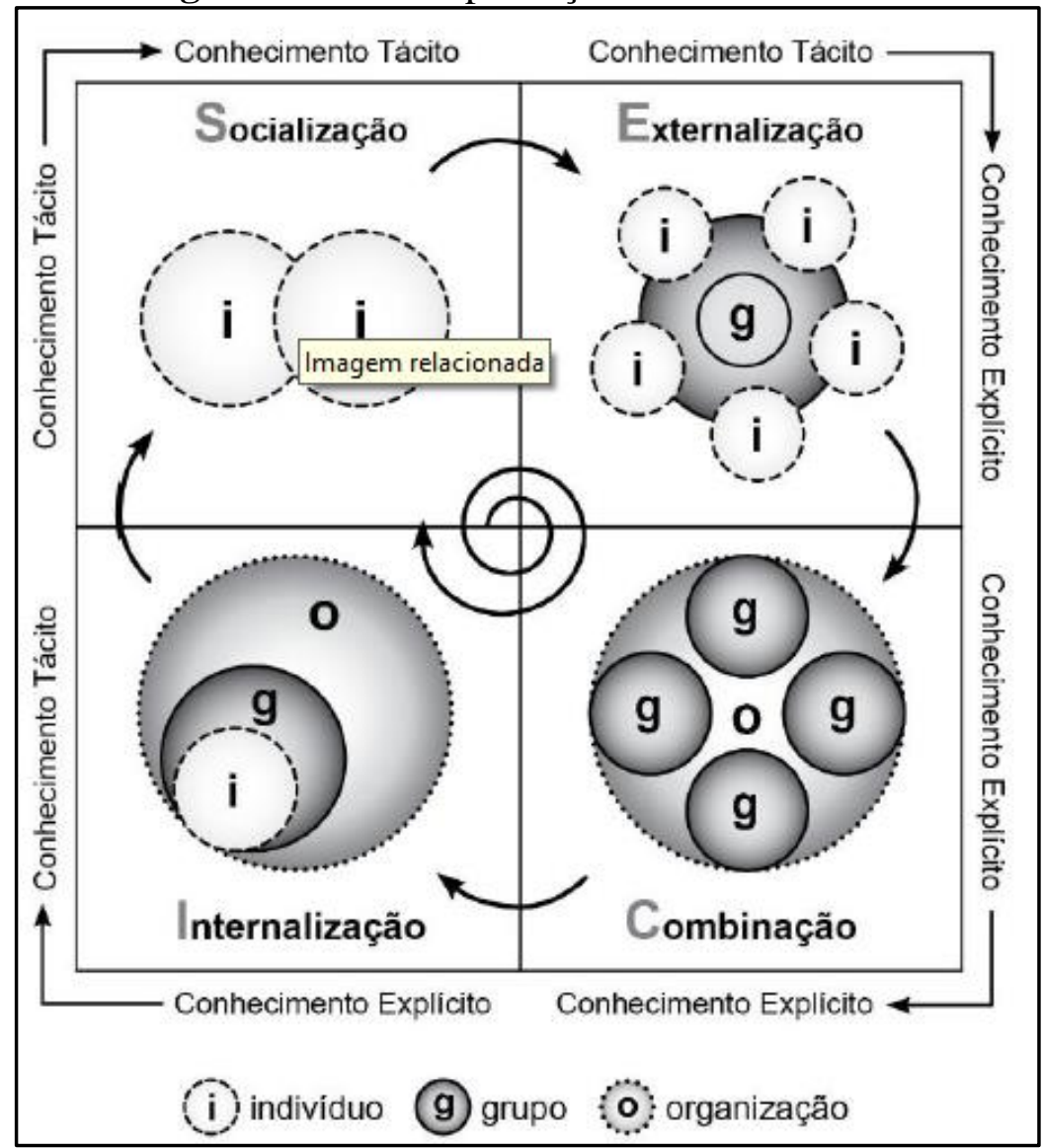

Fonte: Nonaka e Konno (1998)

Esse modelo apresentado é conhecido como espiral do conhecimento e é aplicável nos processos de inovação através das quatro formas de interação existente entre o conhecimento tácito e explícito. Essa espiral do conhecimento inicia-se com a Socialização, momento em 
que há a conversão do conhecimento tácito através de experiências compartilhadas nas interações sociais e técnicas, sendo o mesmo transferido de indivíduo a indivíduo.

Esse conhecimento transmitido individualmente passa a ser compartilhado para grupos através da técnica de Externalização, fazendo com que o conhecimento se torne explícito, compondo uma base conceitual para que exista a produção de novos conhecimentos através de imagens e documentos.

A etapa seguinte do conhecimento é a Combinação, momento em que há a reunião, edição e processamento de conhecimentos explícitos gerando outros conhecimentos explícitos mais complexos que os anteriores e que deverão ser disseminados. Finalizando o processo, a Internalização será a etapa em que o conhecimento explícito, materializado, será aplicado e usado em experiências práticas, passando a compor a base cognitiva para novos processos.

Nesse processo é relevante ressaltar a capacidade de comprometimento necessária dos indivíduos em quererem criar, compartilhar e transferir os conhecimentos, uma vez que sem essa capacidade e disponibilidade o processo não terá sucesso.

\section{Considerações finais}

No presente artigo foi muito citado a questão da eficiência no serviço público, porém cabe ressaltar que em questões legais, a publicidade é um princípio que se faz obrigatório. Cabe ressaltar que a administração pública se preocupa muito em publicar seus atos, seus decretos etc., porém não é comum encontrar manuais de processos e procedimentos de trabalho publicados.

A sociedade exige do Governo que se trabalhe com eficiência para que se consiga atender às suas demandas, que são cada vez maiores, ao contrário dos recursos públicos que estão cada vez mais escassos, seja financeiramente ou em questões de pessoal.

É possível afirmar que a Gestão do Conhecimento possui capacidade de gerar as mudanças necessárias e contribuir para que esse cenário seja modificado, trazendo mais eficiência, eficácia e efetividade às rotinas da administração pública. É necessário que mudanças e aprimoramentos nos processos ocorram de forma sistemática e constante, visando atender aos anseios da população e colaborando para o crescimento do país.

Dessa forma, o conhecimento precisa ser mais bem gerido pelas instituições públicas, seja na criação de manuais, seja na preocupação com a melhoria contínua, revisando seus processos 
frequentemente, seja na disseminação da informação. Enfim, a Gestão do conhecimento é um dos caminhos para melhoria do serviço público e para obtenção da tão sonhada eficiência.

\section{Conclusão}

$\mathrm{O}$ artigo apresentado trouxe aspectos relevantes a toda comunidade, uma vez que procurou introduzir conceitos teóricos sobre administração pública, gestão do conhecimento, inovação e eficiência, podendo, ainda, relacionar esses temas entre si e demonstrar como todos eles devem andar juntos não só na iniciativa privada, nas também nas instituições públicas.

A gestão do conhecimento quando bem desenvolvida e feita com responsabilidade gera qualidade, eficiência, efetividade e desenvolvimento econômico e social, contribuindo para a legalidade, impessoalidade, moralidade e publicidade na administração pública.

Com esse artigo teve com propósito demonstrar que na administração pública em geral, grande parte do conhecimento se encontra em poder de poucos e que na grande maioria das instituições ainda não se possui manual com os procedimentos, portanto o conhecimento se encontra tácito, sendo passado de indivíduo para indivíduo.

A partir de toda a literatura apresentada pude-se perceber que com a gestão correta do conhecimento é possível que se tenha uma disseminação correta do conhecimento institucional o que poderá gerar maior eficiência, redução de ruídos na comunicação e trabalho uniforme entre todos os funcionários, consequentemente aumentando a satisfação daqueles que utilizam o serviço. 


\section{Referências}

ADAM, A.; DELIS, M.; KAMMAS, P. Public sector efficiency: leveling the playing field between OECD countries. Public Choice, 146(1), 163-183, 2011.

ALONSO, José M.; CLIFTON, Judith; DÍAZ-FUENTES, Daniel. The impact of New Public Management on efficiency: An analysis of Madrid's hospitals. Health Policy, [s.1.], v. 119, n. 3, p.333-340, mar. 2015. Elsevier BV. http://dx.doi.org/10.1016/j.healthpol.2014.12.001.

ANDERSSON, Roy et al. Improvement in Public Administration Services: a Case of Business Registration Process. International Journal Of Industrial Engineering And Management, Valencia, v. 9, n. 2, p.109-120, 2018.

ANGELIS, Cristiano Trindade de. Gestão do Conhecimento no setor público: um estudo de caso por meio do método OKA. Revista do Serviço Público, Brasília, v. 62, n. 2, p. 137-166, abr./jun. 2011. Disponível em: https://revista.enap.gov.br/index.php/RSP/article/view/66.

ARANHA, João Gilberto Torres. Plano de desenvolvimento institucional e gestão por competência: percepções de seus desafios por gestores de instituições federais de ensino. 2015. Dissertação (Mestrado em Engenharia de Produção) - Universidade Federal Fluminense, Niterói.

BATISTA, Fábio Ferreira. Modelo de Gestão do Conhecimento para a Administração Pública Brasileira: Como implementar a Gestão do Conhecimento para produzir resultados em benefício do cidadão. Rio de Janeiro: Ipea, 2012. 134 p.

BEM, Roberta Moraes; PRADO Maria Lourdes; DELFINO, Nelson. Desafios à implantação da gestão do conhecimento: a questão cultural nas organizações pública federais brasileiras. Revista Digital de Biblioteconomia e Ciência da Informação. 2013. Disponível em:

http://periodicos.sbu.unicamp.br/ojs/index.php/rdbci/article/view/1641.

BOTELHO, Cássia Regina Ossipe Martins. Gestão do Conhecimento para a melhoria da Gestão Pública: os caminhos da implantação da gestão do conhecimento na Câmara dos Deputados. In: CONGRESSO CONSAD DE GESTÃO PÚBLICA, 2., 2009. Disponível em: http://consad.org.br/evento/ii-congresso/.

BRESSER, L. C. P.. Burocracia pública na construção do Brasil. Versão de junho de 2008. Disponível em: $<$ http://www.bresserpereira.org.br>.

CASTRO, Bruno. A evolução da administração pública. 2010. Disponível em: $<$ https://administradores.com.br/artigos/a-evolucao-da-administracao-publica>.

CEPELOVÁ, Anna; KOREÒOVÁ, Darina. Presumptions of the Process Management Application in Terms of Self-government in the Slovak Republic. Revista Administratie Si Management Public, Romania, v. 28, p.133-152, 2017.

CHIECHELSKI, P. C. S.. Avaliação de programas sociais: abordagens quantitativas e suas limitações. Textos \& Contextos, 4(1), 1-12., 2005.

CICCIO, Claudio di; MARRELLA, Andrea; RUSSO, Alessandro. Knowledge-Intensive Processes: Characteristics, Requirements and Analysis of Contemporary Approaches. Journal On Data Semantics, [s.1.], v. 4, n. 1, p.29-57, 9 abr. 2014. Springer Science and Business Media LLC. http://dx.doi.org/10.1007/s13740014-0038-4.

COUTO, Gilberto de Araújo Brandão et al. Systematic proposal for improving the fuel supply surveillance in Brazil: Application to a real case. Revista Gestão Inovação e Tecnologias, [s.1.], v. 8, n. 4, p.4588-4602, 15 dez. 2018. Associacao Academica de Propriedade Intelectual. http://dx.doi.org/10.7198/geintec.v8i4.1304.

DI PIETRO, Maria Sylvia Zanella. Direito administrativo. 25 ed. São Paulo: Atlas, 2012. 
FADUL, Élvia Mirian Cavalcanti; SILVA, Lindomar Pinto da. Retomando o debate sobre a Reforma do Estado e a Nova Administração Pública. In: ENCONTRO NACIONAL DE PÓS GRADUAÇÃO E PESQUISA EM ADMINISTRAÇÃO - ANPAD, 2008, Rio de Janeiro/RJ.

FERRARI, Alessio et al. Improving the quality of business process descriptions of public administrations. Business Process Management Journal, [s.1.], v. 24, n. 1, p.49-66, 2 fev. 2018. Emerald. http://dx.doi.org/10.1108/bpmj-05-2016-0096.

FRESNEDA, Paulo Sérgio Vilches; GONÇALVES, Sonia Maria Goulart. A Experiência Brasileira na Formulação de uma Proposta de Política de Gestão do Conhecimento para a Administração Pública Federal. Câmara dos Deputados, Brasília, 2007. Disponível em: http://www.bibl.ita.br/PoliticaGC.pdf.

JACOBSEN, Alessandra de Linhares; MORETTO NETO, Luís. Teorias da administração II. Florianópolis: Departamento de Ciências da Administração / UFSC; [Brasília]: CAPES, 2009. 170 p. Disponível em: < https://canal.cecierj.edu.br/012016/4efa83217ac00f6b1d722c96eb >.

JUÁREZ, Hernándesz José Luis; CERVANTES, Baltazar Pérez. La gestión del conocimiento como estratégia para la mejora continua em la Administración Publica Municipal. La experiencia del H Ayuntamiento de Novolato. Internacional Jounal of Good Conscience, v. 7, n. 3, p. 1-14. nov./2012. Disponível em: http://www.spentamexico.org/v7-n3/7(3)1-14.pdf.

LIMA, Tharcísio Pimentel. A importância da Eficiência e Eficácia na Gestão Pública. 2014. Disponível em: $<$ https://www.portaleducacao.com.br/conteudo/artigos/administracao/a-importancia-da-eficiencia-e-eficacia-nagestao-publica/55602>.

MEIRELLES, Hely Lopes. Direito administrativo brasileiro. 23 ed. atual. São Paulo: Malheiros, 1998.

MIRANDA, Lara Caxico Martins; KEMPFER, Marlene. Adminnistração pública gerencial brasileira e os desafios para prosseguir na sua implementação. Revista do Direito Público, [s.1.], v. 13, n. 3, p.130-152, 23 dez. 2018. Universidade Estadual de Londrina. http://dx.doi.org/10.5433/1980-511x.2018v13n3p130.

MULGAN, G.; ALBURY, D. Innovation in the Public Sector. London: Cabinet Office Strategy Unit, 2003. Disponível em: 〈file:///D:/Downloads/innovation-in-the-public-sector\%20(1).pdf〉.

NONAKA, I., KONNO, N. The Concept of "Ba': Building Foundation for Knowledge Creation. California Management Review, 40, 3. Spring, 1998.

NONAKA, I.; TAKEUCHI, H. Criação de conhecimento na empresa. Tradução Ana Beatriz Rodrigues. 13. ed. Rio de Janeiro: Elsevier, 1997.

NOVOA, Viviana Duro. Approach to the Application of Business Process Management in the Cuban Public Administration. Gecontec: Revista Internacional de Gestión del Conocimiento y La Tecnología., Havana, v. 6, n. 1, p.59-67, 2018.

PAES DE PAULA, A. P. Por uma nova gestão pública: limites e potencialidades da experiência contemporânea. Rio de Janeiro: Fundação Getúlio Vargas, 2005.

PARDO, Sebastián; CORONEL, Juan Enrique; BERTONE, Rodolfo; THOMAS, Pablo. Gestión del Conocimiento: um enfoque aplicado em la Administración Pública. Argentina, 2013.

SANTOS, Vanessa dos; BASTOS, Rogério Cid, 2019. Avaliação da Maturidade da Gestão do Conhecimento na Administração Pública. Perspectivas em Gestão \& Conhecimento, v. 9, n. 1, pp. 24-41, jan./abr. 2019. DOI: http://dx.doi.org/10.21714/2236-417X2019v9n1p24.

STASTNA, L.; GREGOR, M. Local government efficiency: evidence from the Czech municipalities (IES Working Paper No. 14, 2011). Prague, Czech Republic: Charles University, 2011. 